\title{
Comparison of Fixed Point Methods and Krylov Subspace Methods Solving Convection-Diffusion Equations
}

\author{
Xijian Wang \\ School of Mathematics and Computational Science, Wuyi University, Jiangmen, China \\ Email: wangxj1980426@163.com
}

Received 2 March 2015; accepted 5 June 2015; published 9 June 2015

Copyright (C) 2015 by author and Scientific Research Publishing Inc.

This work is licensed under the Creative Commons Attribution International License (CC BY).

http://creativecommons.org/licenses/by/4.0/

(c) () Dpen Access

\section{Abstract}

The paper first introduces two-dimensional convection-diffusion equation with boundary value condition, later uses the finite difference method to discretize the equation and analyzes positive definite, diagonally dominant and symmetric properties of the discretization matrix. Finally, the paper uses fixed point methods and Krylov subspace methods to solve the linear system and compare the convergence speed of these two methods.

\section{Keywords}

Finite Difference Method, Convection-Diffusion Equation, Discretization Matrix, Iterative Method, Convergence Speed

\section{Introduction}

In the case of a linear system $A x=b$, the two main classes of iterative methods are the stationary iterative methods (fixed point methods) [1]-[3], and the more general Krylov subspace methods [4]-[13]. When these two classical iterative methods suffer from slow convergence for problems which arise from typical applications such as fluid dynamics or electronic device simulation, preconditioning [14]-[16] is a key ingredient for the success of the convergent process.

The goal of this paper is to find an efficient iterative method combined with preconditioning for the solution of the linear system $A x=b$ which is related to the following two-dimensional boundary value problem (BVP) [17]: 


$$
\begin{cases}-\left(\frac{\partial^{2} u}{\partial x^{2}}+\frac{\partial^{2} u}{\partial y^{2}}\right)+\gamma\left(\frac{\partial u}{\partial x}-\frac{\partial u}{\partial y}\right)=f(x, y), & (x, y) \in \Omega=(0,1)^{2} \\ u=0, & (x, y) \in \partial \Omega .\end{cases}
$$

For parameter $\gamma=0,16,64$, we use finite difference method to discretize the Equation (1). Take $n+2$ points in both the $x$ - and $y$-direction and number the related degrees of freedom first left-right and next bottomtop. Now, we take $n=32$, thus the grid size $\Delta x=\Delta y=h=\frac{1}{n+2-1}=\frac{1}{33}$. For $\gamma=0$, the convection term equals to 0 , using central difference method to the diffusion term, we get the discretization matrix of the Equation (1)

$$
A_{0}=\left(\begin{array}{ccccc}
B_{0} & -I & O & \cdots & O \\
-I & B_{0} & -I & \ddots & \vdots \\
O & \ddots & \ddots & \ddots & O \\
\vdots & \ddots & -I & B_{0} & -I \\
O & O & O & -I & B_{0}
\end{array}\right), B_{0}=\left(\begin{array}{ccccc}
4 & -1 & 0 & \cdots & 0 \\
-1 & 4 & -1 & \ddots & \vdots \\
0 & \ddots & \ddots & \ddots & 0 \\
\vdots & \ddots & -1 & 4 & -1 \\
0 & 0 & 0 & -1 & 4
\end{array}\right) .
$$

For $\gamma=16$, the diffusion term use a central difference and for the convection term use central differences scheme as the following:

$$
f\left(x_{i j}, y_{i j}\right)=-\left(\frac{u_{(i+1) j}-2 u_{i j}+u_{(i-1) j}}{h^{2}}+\frac{u_{i(j+1)}-2 u_{i j}+u_{i(j-1)}}{h^{2}}\right)+\gamma\left(\frac{u_{(i+1) j}-u_{(i-1) j}}{2 h}-\frac{u_{i(j+1)}-u_{i(j-1)}}{2 h}\right),
$$

we obtain the discretization matrix of the Equation (1)

$$
A_{1}=\left(\begin{array}{ccccc}
B_{1} & -\left(1+c_{1}\right) I & O & \cdots & O \\
-\left(1-c_{1}\right) I & B_{1} & -\left(1+c_{1}\right) I & \ddots & \vdots \\
O & \ddots & \ddots & \ddots & O \\
\vdots & \ddots & -\left(1-c_{1}\right) I & B_{1} & -\left(1+c_{1}\right) I \\
O & O & O & -\left(1-c_{1}\right) I & B_{1}
\end{array}\right),
$$

where

$$
B_{1}=\left(\begin{array}{ccccc}
4 & -\left(1-c_{1}\right) & 0 & \cdots & 0 \\
-\left(1+c_{1}\right) & 4 & -\left(1-c_{1}\right) & \ddots & \vdots \\
0 & \ddots & \ddots & \ddots & 0 \\
\vdots & \ddots & -\left(1+c_{1}\right) & 4 & -\left(1-c_{1}\right) \\
0 & 0 & 0 & -\left(1+c_{1}\right) & 4
\end{array}\right), c_{1}=\frac{\gamma h}{2}=\frac{8}{33} .
$$

For $\gamma=64$, the diffusion term use a central difference and for the convection term use upwind differences scheme as the following:

$$
f\left(x_{i j}, y_{i j}\right)=-\left(\frac{u_{(i+1) j}-2 u_{i j}+u_{(i-1) j}}{h^{2}}+\frac{u_{i(j+1)}-2 u_{i j}+u_{i(j-1)}}{h^{2}}\right)+\gamma\left(\frac{u_{i j}-u_{(i-1) j}}{h}-\frac{u_{i(j+1)}-u_{i j}}{h}\right),
$$

we obtain the discretization matrix of the Equation (1)

$$
A_{2}=\left(\begin{array}{ccccc}
B_{2} & -\left(1+c_{1}\right) I & O & \cdots & O \\
-I & B_{2} & -\left(1+c_{1}\right) I & \ddots & \vdots \\
O & \ddots & \ddots & \ddots & O \\
\vdots & \ddots & -I & B_{2} & -\left(1+c_{1}\right) I \\
O & O & O & -I & B_{2}
\end{array}\right)
$$


where

$$
B_{2}=\left(\begin{array}{ccccc}
4+2 c_{2} & -1 & 0 & \cdots & 0 \\
-\left(1+c_{1}\right) & 4+2 c_{2} & -1 & \ddots & \vdots \\
0 & \ddots & \ddots & \ddots & 0 \\
\vdots & \ddots & -\left(1+c_{1}\right) & 4+2 c_{2} & -1 \\
0 & 0 & 0 & -\left(1+c_{1}\right) & 4+2 c_{2}
\end{array}\right), c_{2}=\gamma h=\frac{64}{33}
$$

\section{Properties of the Discretization Matrix}

In this section, we would first compute the eigenvalues of the discretization matrices $A_{0}, A_{1}$ and $A_{2}$ of Equation (1), later analyze positive definite, diagonally dominant and symmetric properties of these matrices.

\subsection{Eigenvalues}

Using MATLAB, the eigenvalues of the discretization matrices $A_{0}, A_{1}$ and $A_{2}$ of Equation (1) are the following:

$$
\left\{\begin{array}{l}
\lambda\left(A_{0}\right)_{p, q}=4+2\left(\cos \left(\frac{p \pi}{n+1}\right)+\cos \left(\frac{q \pi}{n+1}\right)\right) \\
\lambda\left(A_{1}\right)_{p, q}=4+2 \sqrt{1-c_{1}^{2}}\left(\cos \left(\frac{p \pi}{n+1}\right)+\cos \left(\frac{q \pi}{n+1}\right)\right), \quad p, q=1,2, \cdots, n . \\
\lambda\left(A_{2}\right)_{p, q}=4+2 c_{2}+2 \sqrt{1+c_{2}}\left(\cos \left(\frac{p \pi}{n+1}\right)+\cos \left(\frac{q \pi}{n+1}\right)\right)
\end{array}\right.
$$

\subsection{Definite Positiveness}

Matrix $A$ is positive definite if and only if the symmetric part of $A$ i.e. $A_{s}=\frac{A+A^{\mathrm{T}}}{2}$ is positive definite. Since

$$
\left(A_{i}\right)_{S}=\left\{\begin{array}{lr}
A_{0}, & i=0 \\
A_{0}, & i=1 \\
\left(1+\frac{c_{2}}{2}\right) A_{0}, & i=2
\end{array}\right.
$$

we have all the eigenvalues of $\frac{A_{i}+A_{i}^{\mathrm{T}}}{2}(i=0,1,2)$ are the following:

$\lambda\left(\left(A_{i}\right)_{S}\right)_{p, q}=\left\{\begin{array}{l}\lambda\left(A_{0}\right)_{p, q}=4+2 \sqrt{1-c_{1}^{2}}\left(\cos \left(\frac{p \pi}{n+1}\right)+\cos \left(\frac{q \pi}{n+1}\right)\right)>0, \quad i=0,1, \\ \lambda\left(\left(1+\frac{c_{2}}{2}\right) A_{0}\right)_{p, q}=\left(1+\frac{c_{2}}{2}\right)\left(4+2 \sqrt{1-c_{1}^{2}}\left(\cos \left(\frac{p \pi}{n+1}\right)+\cos \left(\frac{q \pi}{n+1}\right)\right)\right)>0, i=2,\end{array} \quad p, q=1,2, \cdots, n\right.$.

Therefore $\left(A_{i}\right)_{S} \quad(i=0,1,2)$ is positive definite, which means the discretization matrices $A_{0}, A_{1}$ and $A_{2}$ of Equation (1) are positive definite.

\subsection{Diagonal Dominance}

For all the discretization matrices $A_{0}, A_{1}$ and $A_{2}$ of Equation (1), 


$$
\sum_{i \neq j, i=1}^{n}\left|a_{i j}\right| \leq \begin{cases}2+2=4=\left|a_{j j}\right|, & A_{0}=\left(a_{i j}\right) ; \\ 2(1+c)+2(1-c)=4=\left|a_{i j}\right|, & A_{1}=\left(a_{i j}\right) ; \\ 2(1+c)+2=4+2 c=\left|a_{j j}\right|, & A_{2}=\left(a_{i j}\right) .\end{cases}
$$

Therefore, the discretization matrices $A_{0}, A_{1}$ and $A_{2}$ of Equation (1) are diagonally dominant.

\subsection{Symmetriness}

It is easy to see that only $A_{0}$ is symmetric.

\section{Stationary Iteration Methods and Krylov Subspace Methods}

The goal of this section is to find an efficient iterative method for the solution of the linear system $A_{i} x=b, i=0,1,2$. Since $A_{i}(i=0,1,2)$ are positive definite and diagonally dominant, we would use fixed point methods and Krylov subspace methods. In this section, first find the suitable convergence tolerance, later use numerical experiments to compare the convergence speed of various iteration methods.

\subsection{Convergence Tolerance}

Without loss of generality, take $b=A x_{*}, x_{*}=(1,1, \cdots, 1)^{\mathrm{T}}$, convergence tolerance $\delta=\frac{10^{-3}}{\|b\|_{2} \cdot\left\|A_{i}^{-1}\right\|_{2}}$ and assume that $\frac{\left\|A x_{i}-b\right\|_{2}}{\|b\|_{2}} \leq \delta$, then

$$
\begin{aligned}
\left\|x_{i}-x_{*}\right\|_{2} & =\left\|A^{-1} \cdot\left(A x_{i}-b\right)\right\|_{2} \leq\left\|A^{-1}\right\|_{2} \cdot\left\|A x_{i}-b\right\|_{2} \\
& =\|b\|_{2} \cdot\left\|A^{-1}\right\|_{2} \cdot\left(\frac{\left\|A x_{i}-b\right\|_{2}}{\|b\|_{2}}\right) \leq \delta \cdot\|b\|_{2} \cdot\left\|A^{-1}\right\|_{2}=10^{-3} .
\end{aligned}
$$

In order to achieve $\left\|x_{i}-x_{*}\right\|_{2} \leq 10^{-3}$ in the numerical experiments, we would set convergence tolerance $\delta=\frac{10^{-3}}{\|b\|_{2} \cdot\left\|A_{i}^{-1}\right\|_{2}}$.

\subsection{Numerical Experiments}

Computational results using fixed point methods such as Jacobi, Gauss-Seidel, SOR etc. and projection methods such as PCG, BICG, BICGSTAB, CGS, GMRES and QMR are listed out in figures (Figures 1-21), for all three different $\gamma$ values $(\gamma=0,16,64)$. The projection methods are performed with different preconditioning methods such as Jacobi preconditioning, luinc and cholinc preconditioning.

The tables (Table 1 and Table 2) containing relevant computational details are also given below.

\section{Conclusions}

From the figures (Figures 1-21) and tables (Table 1 and Table 2), we obtain the following conclusions:

- The convergence speeds of SOR, Backward SOR and SSOR are faster than that of GS and Backward GS; while GS and Backward GS are faster than Jacobi;

- If matrix A is symmetric $(\gamma=0)$, the convergence speeds of SOR, Backward SOR and SSOR are the same; otherwise $(\gamma=16,64)$, SSOR is faster than Backward SOR and SOR;

- The convergence speeds of SOR and Backward SOR are the same, also for GS and Backward GS;

- From Table 2, the iteration steps and the time for all fixed point methods of the case in which $\gamma=64$ are less than the case in which $\gamma=16$, and also the case in which $\gamma=16$ are less than the case in which $\gamma=0$;

- The upwind difference method is more suitable to be applied to convection dominant problem than the cen- 


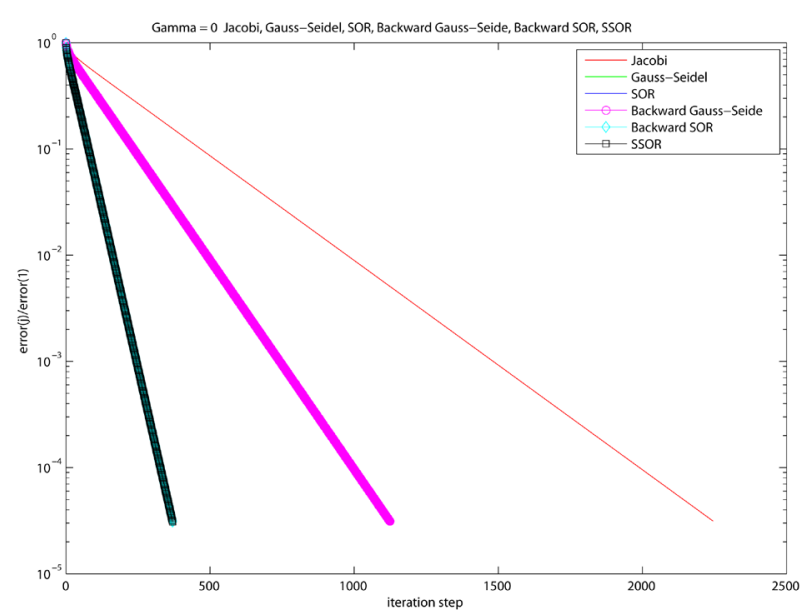

Figure 1. Comparison of convergence speed using fixed point methods when $\gamma=0$.

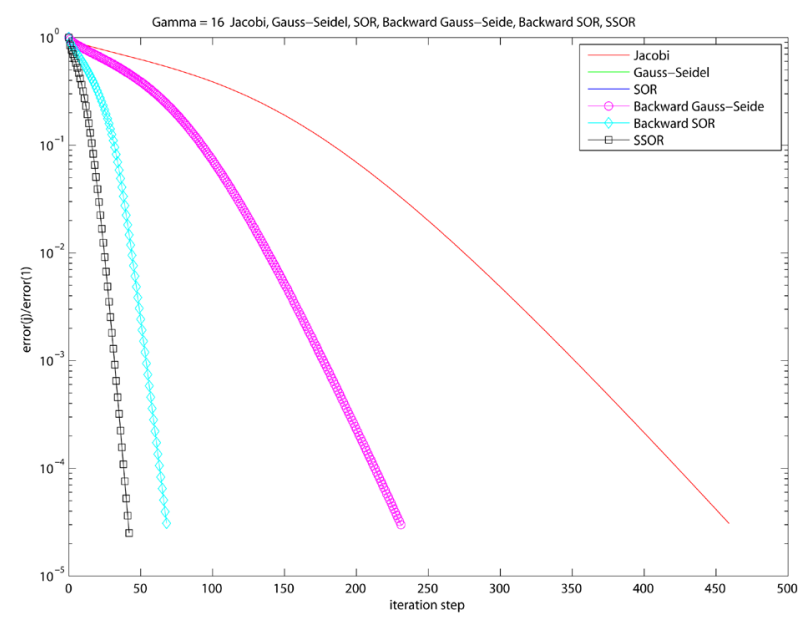

Figure 2. Comparison of convergence speed using fixed point methods when $\gamma=16$.

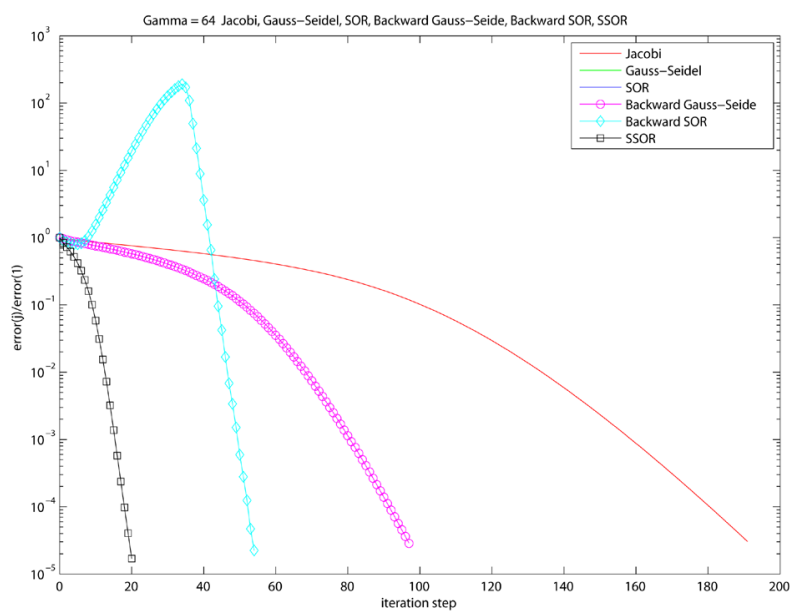

Figure 3. Comparison of convergence speed using fixed point methods when $\gamma=64$. 


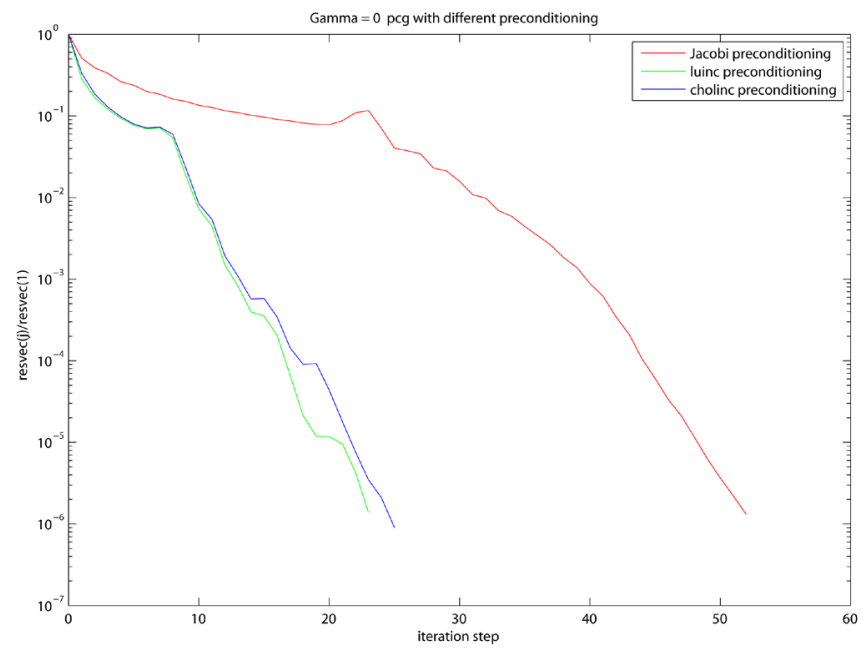

Figure 4. PCG with different preconditionings when $\gamma=0$.

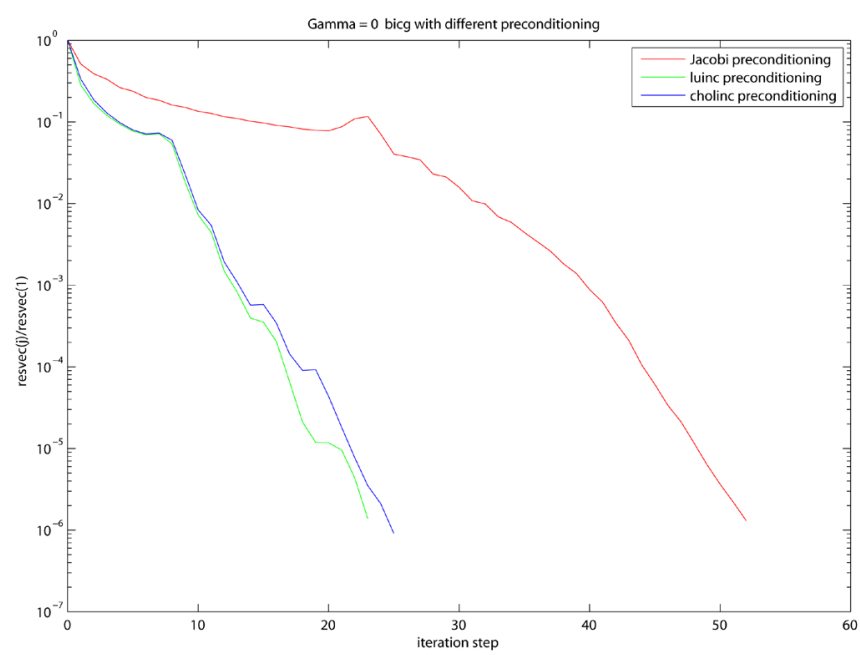

Figure 5. BICG with different preconditionings when $\gamma=0$.

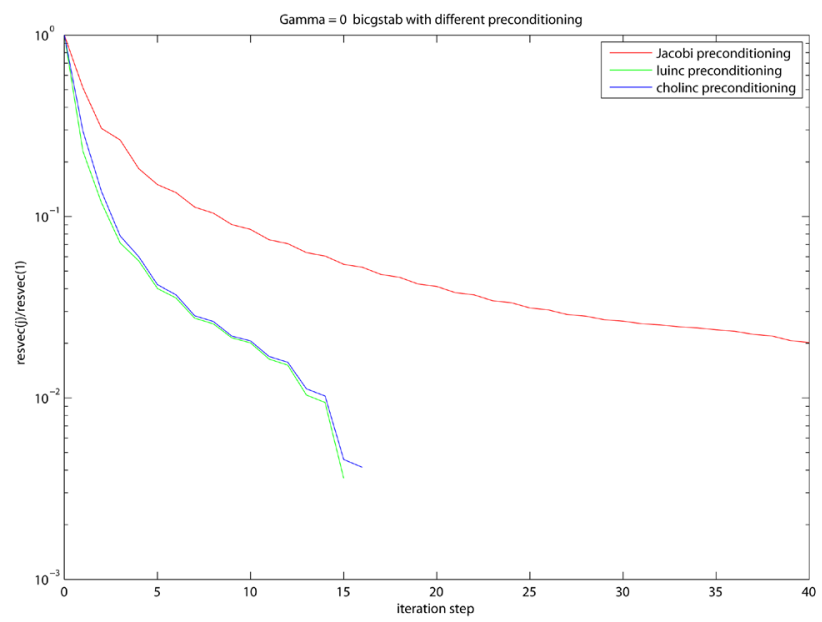

Figure 6. BICGSTAB with different preconditionings when $\gamma=0$. 


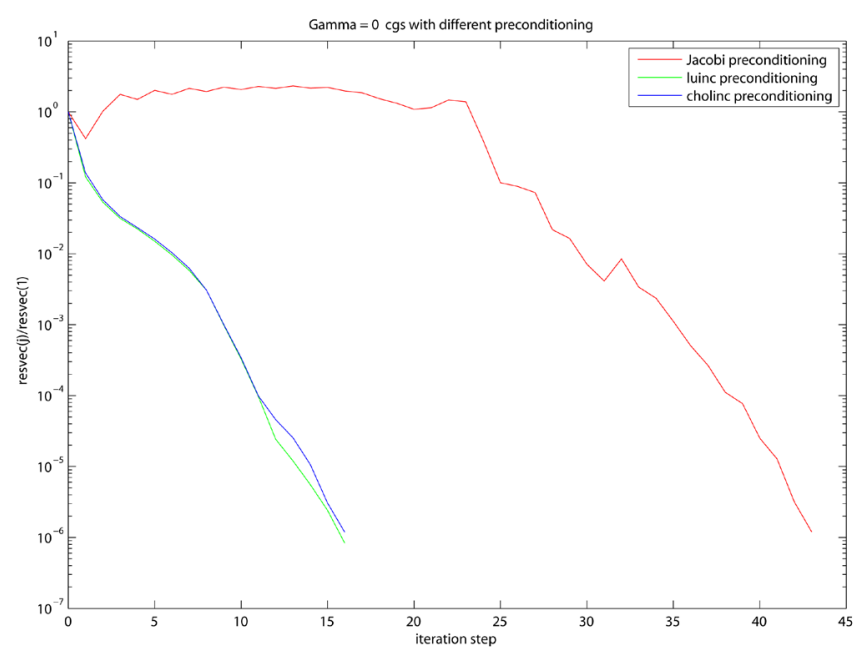

Figure 7. CGS with different preconditionings when $\gamma=0$.

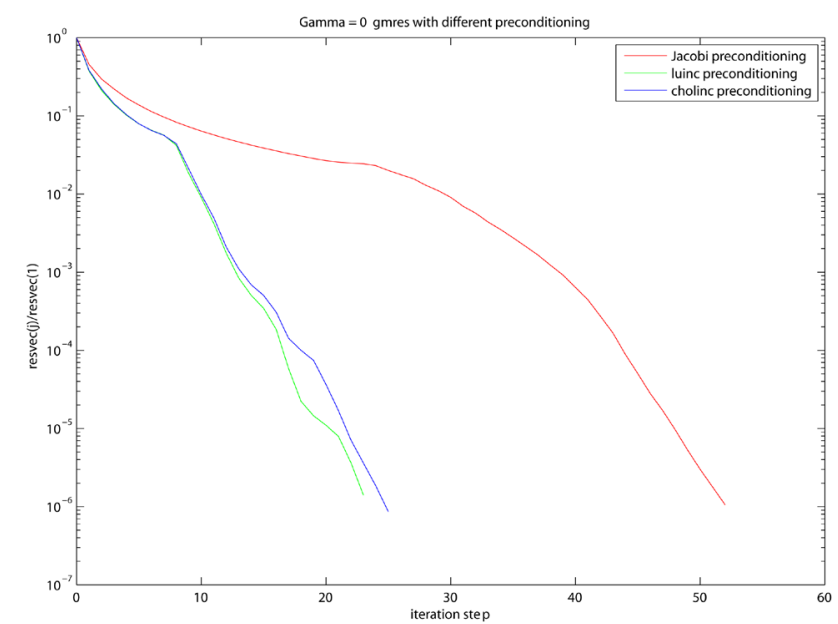

Figure 8. GMRES with different preconditionings when $\gamma=0$.

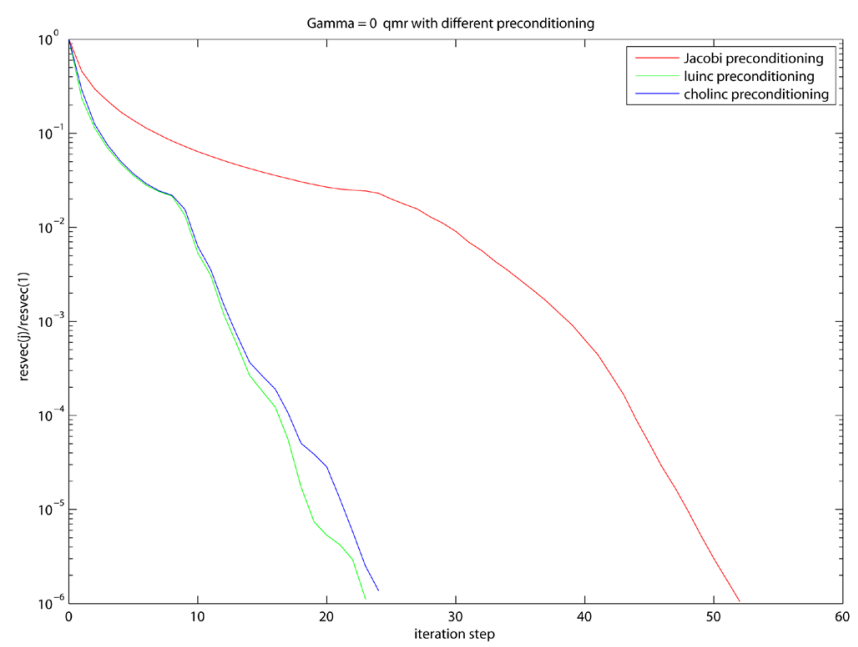

Figure 9. QMR with different preconditionings when $\gamma=0$. 


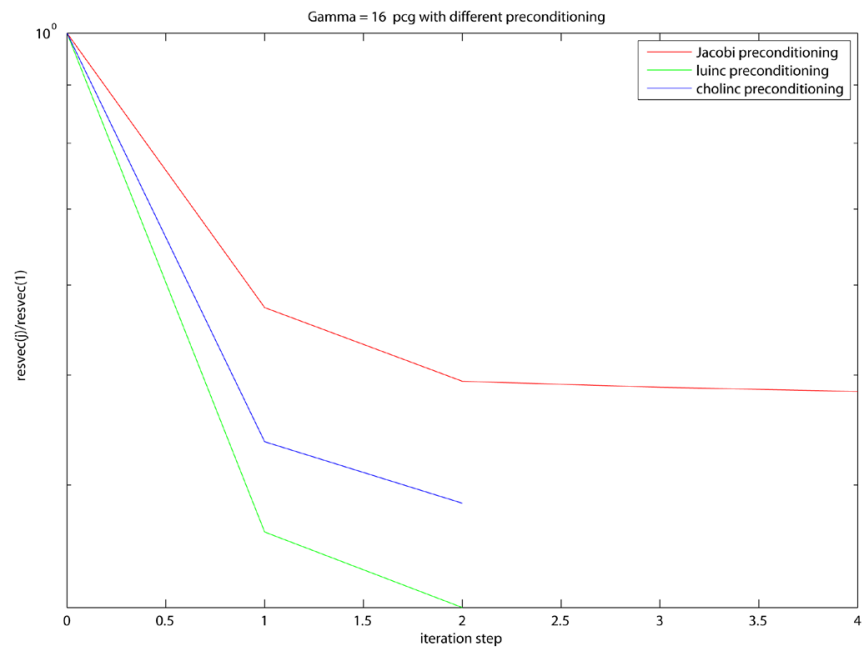

Figure 10. PCG with different preconditionings when $\gamma=16$.

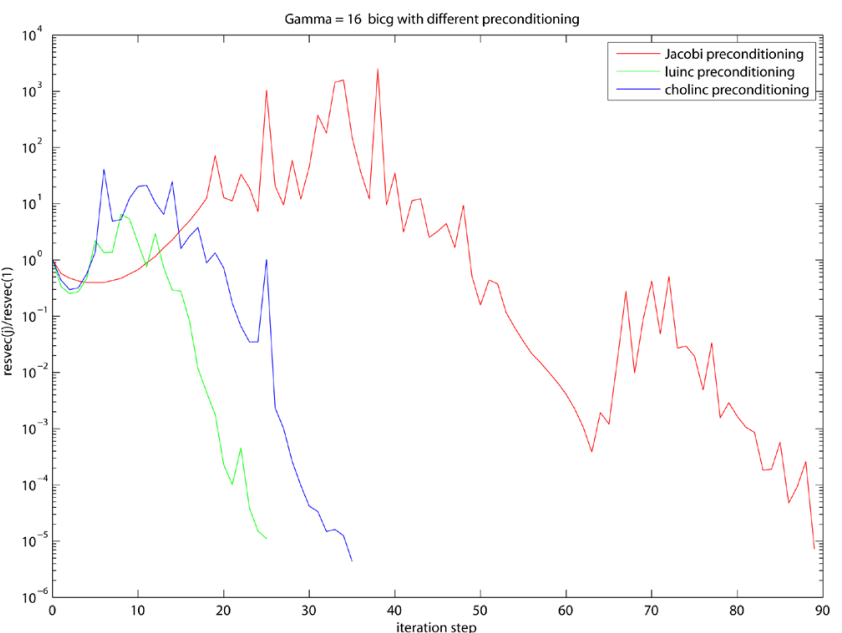

Figure 11. BICG with different preconditionings when $\gamma=16$.

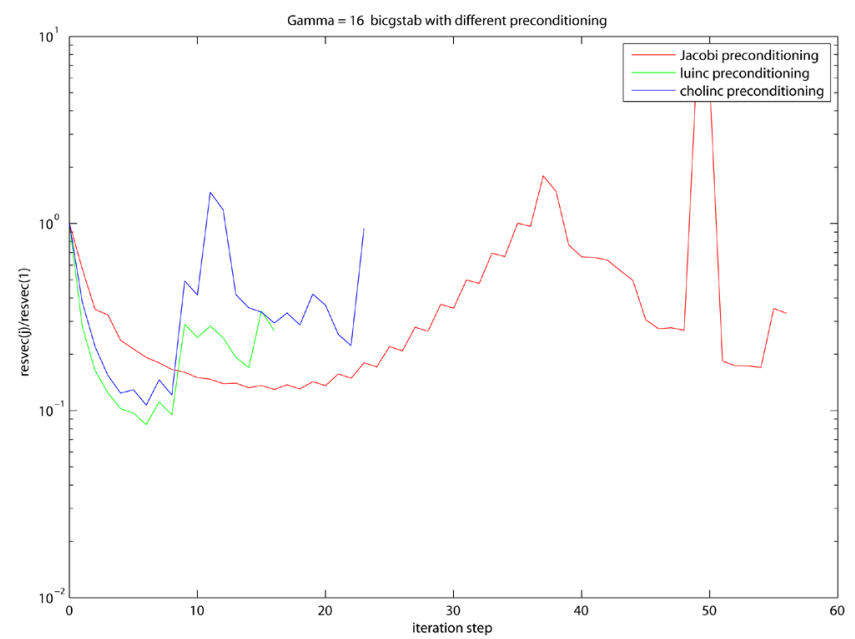

Figure 12. BICGSTAB with different preconditionings when $\gamma=16$. 


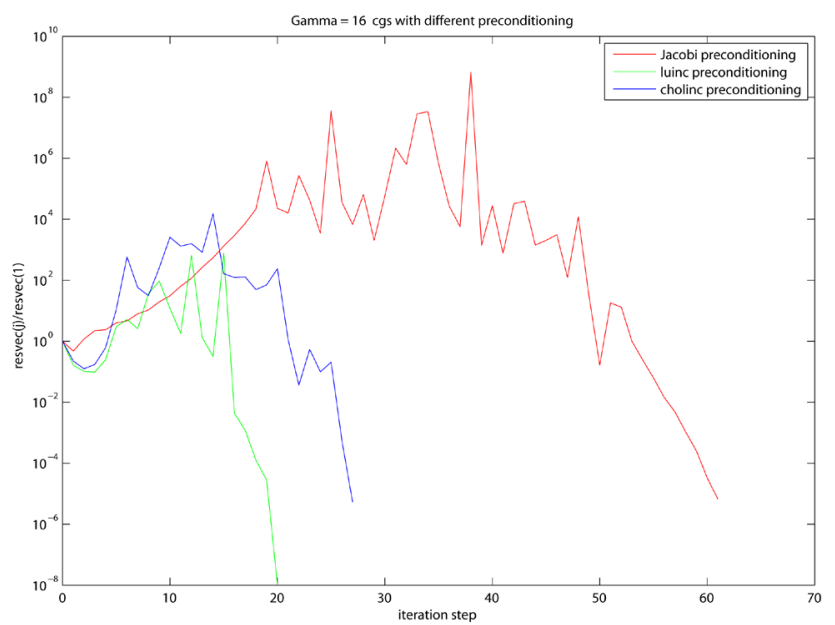

Figure 13. CGS with different preconditionings when $\gamma=16$.

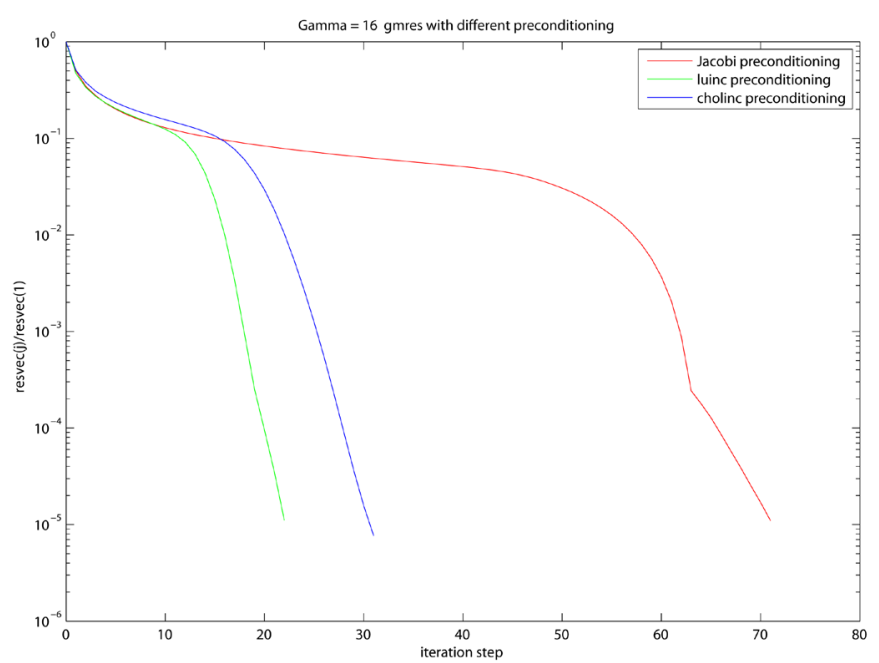

Figure 14. GMRES with different preconditionings when $\gamma=16$.

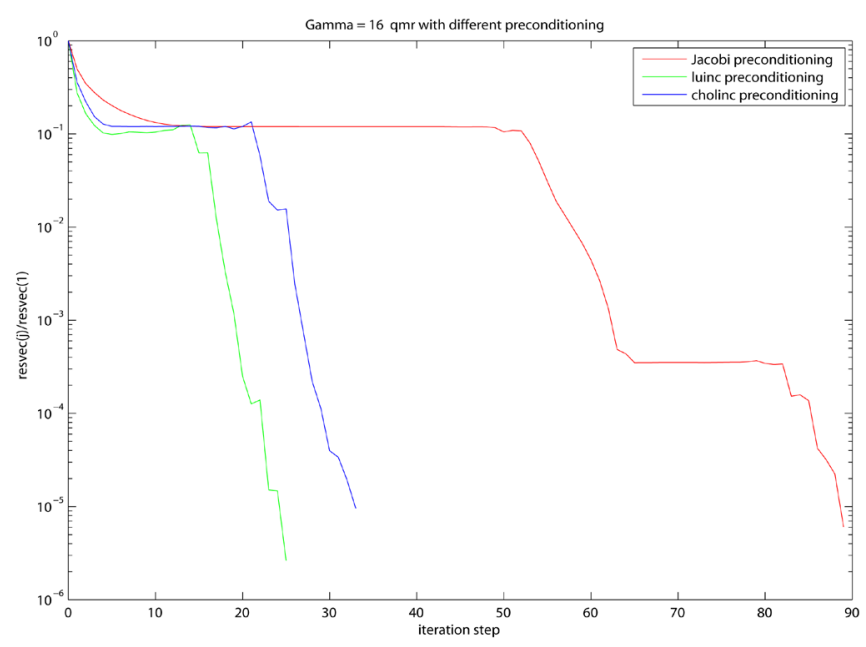

Figure 15. QMR with different preconditionings when $\gamma=16$. 


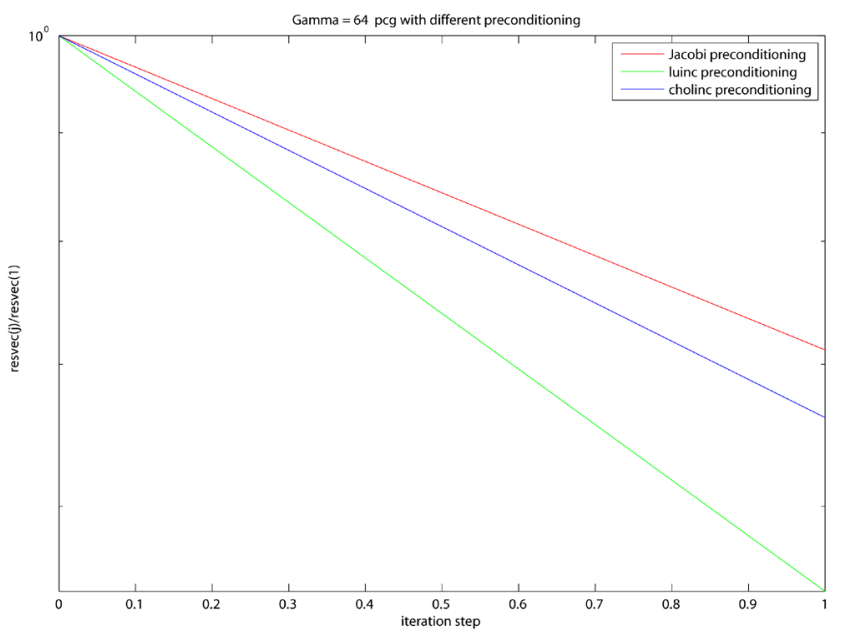

Figure 16. PCG with different preconditionings when $\gamma=64$.

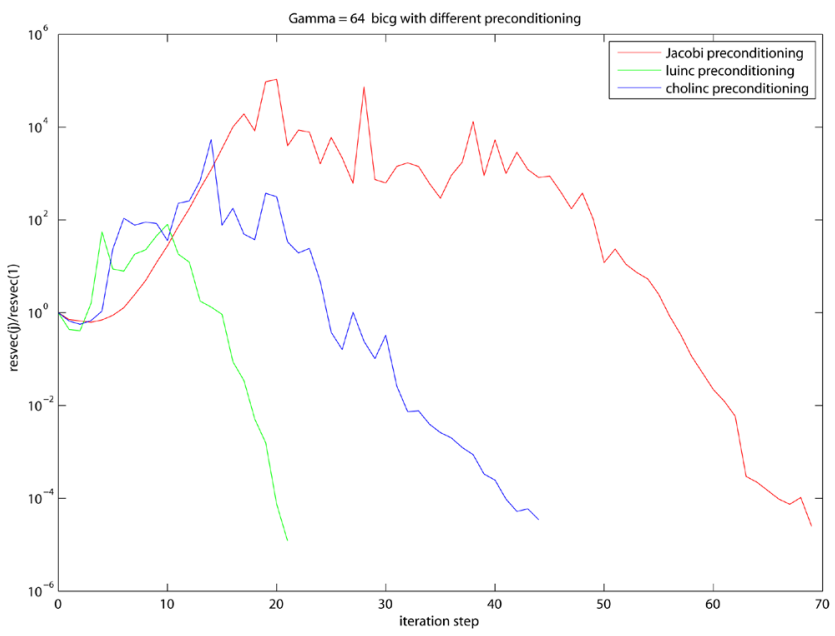

Figure 17. Bicg with different preconditionings when $\gamma=64$.

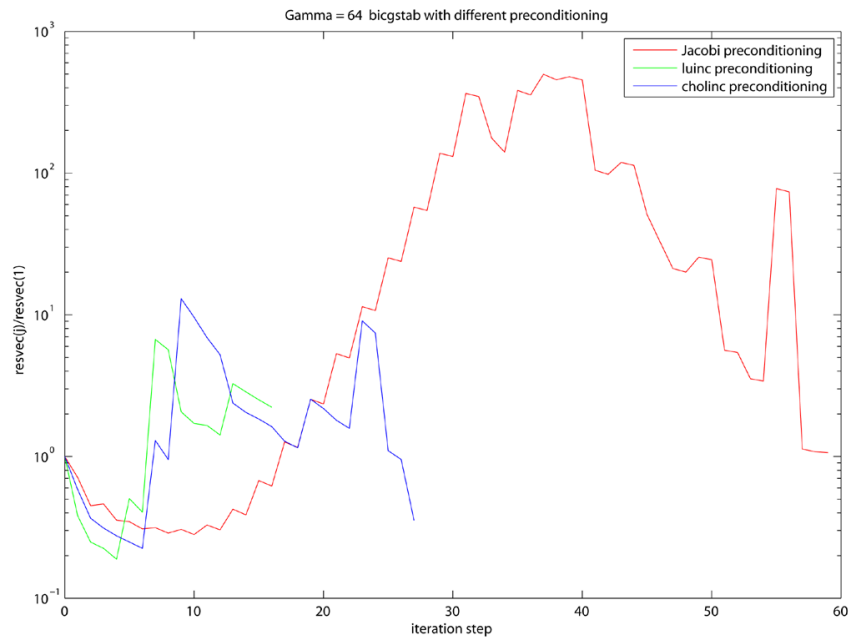

Figure 18. BICGSTAB with different preconditionings when $\gamma=64$. 


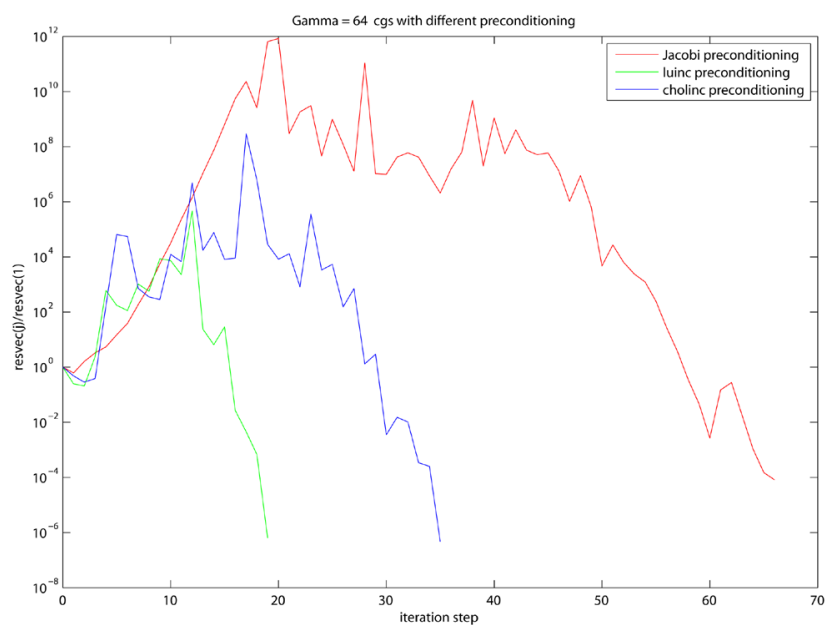

Figure 19. CGS with different preconditionings when $\gamma=64$.

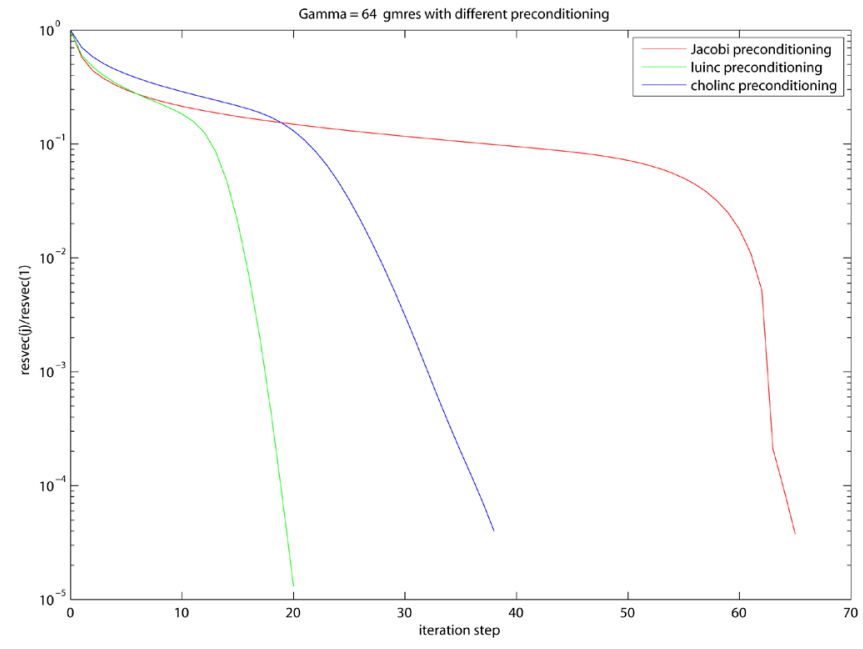

Figure 20. GMRES with different preconditionings when $\gamma=64$.

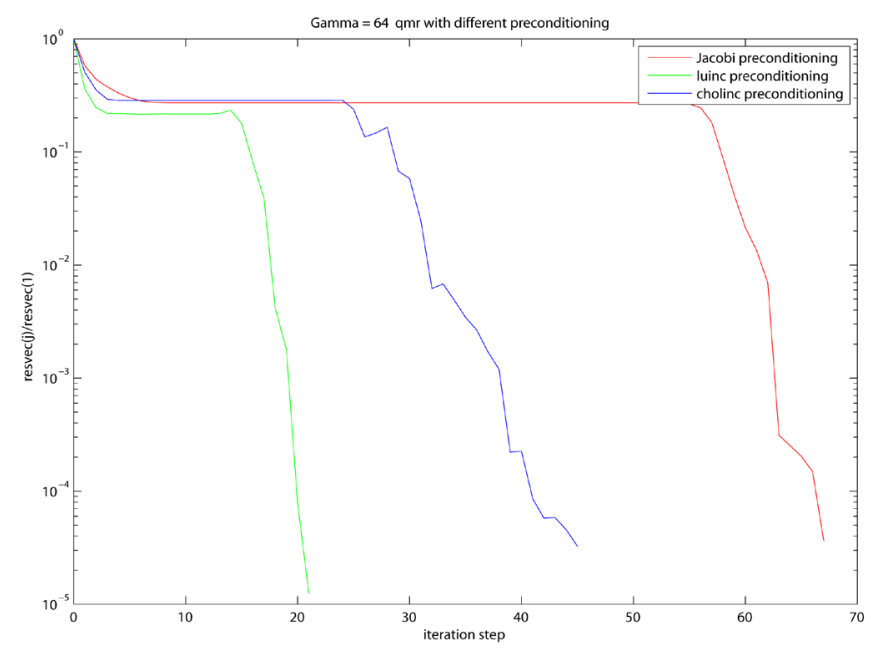

Figure 21. QMR with different preconditionings when $\gamma=64$. 
Table 1. Computational details of different projection methods with different preconditioning.

\begin{tabular}{|c|c|c|c|c|c|c|}
\hline Gamma & Function & Preconditioning & flag & No. of iterations & Relres & Delta (tol) \\
\hline \multirow{27}{*}{0} & \multirow{3}{*}{ PCG } & jacobi & 0 & 52 & $1.31 \mathrm{E}-06$ & \multirow[t]{18}{*}{$1.55 \mathrm{E}-06$} \\
\hline & & luinc & 0 & 23 & $1.38 \mathrm{E}-06$ & \\
\hline & & cholinc & 0 & 25 & $9.05 \mathrm{E}-07$ & \\
\hline & \multirow{3}{*}{ BICG } & jacobi & 0 & 52 & $1.31 \mathrm{E}-06$ & \\
\hline & & luinc & 0 & 23 & $1.38 \mathrm{E}-06$ & \\
\hline & & cholinc & 0 & 25 & $9.05 \mathrm{E}-07$ & \\
\hline & \multirow{3}{*}{ BICGSTAB } & jacobi & 0 & 40.5 & $1.42 \mathrm{E}-06$ & \\
\hline & & luinc & 0 & 15.5 & $9.36 \mathrm{E}-07$ & \\
\hline & & cholinc & 0 & 16 & $1.54 \mathrm{E}-06$ & \\
\hline & \multirow{3}{*}{ CGS } & jacobi & 0 & 43 & $1.20 \mathrm{E}-06$ & \\
\hline & & luinc & 0 & 16 & $8.27 \mathrm{E}-07$ & \\
\hline & & choline & 0 & 16 & $1.18 \mathrm{E}-06$ & \\
\hline & \multirow{3}{*}{ GMRES } & jacobi & 0 & 49 & $1.32 \mathrm{E}-06$ & \\
\hline & & luinc & 0 & 23 & $9.01 \mathrm{E}-07$ & \\
\hline & & choline & 0 & 24 & $1.21 \mathrm{E}-06$ & \\
\hline & \multirow{3}{*}{ QMR } & jacobi & 0 & 52 & $1.05 \mathrm{E}-06$ & \\
\hline & & luinc & 0 & 23 & $1.12 \mathrm{E}-06$ & \\
\hline & & choline & 0 & 24 & $1.37 \mathrm{E}-06$ & \\
\hline & \multirow{3}{*}{ PCG } & jacobi & 1 & 4 & 0.483607757 & \multirow[t]{18}{*}{$1.14 \mathrm{E}-05$} \\
\hline & & luinc & 1 & 2 & 0.312145391 & \\
\hline & & choline & 1 & 2 & 0.38563672 & \\
\hline & & jacobi & 0 & 89 & $7.33 \mathrm{E}-06$ & \\
\hline & bicg & luinc & 0 & 25 & $1.11 \mathrm{E}-05$ & \\
\hline & & choline & 0 & 35 & $4.37 \mathrm{E}-06$ & \\
\hline & & jacobi & 0 & 56.5 & $1.03 E-05$ & \\
\hline & BICGSTAB & luinc & 0 & 16.5 & $6.30 \mathrm{E}-06$ & \\
\hline & & choline & 0 & 23.5 & $3.98 \mathrm{E}-06$ & \\
\hline \multirow[t]{9}{*}{16} & \multirow{3}{*}{ CGS } & jacobi & 0 & 61 & $6.61 \mathrm{E}-06$ & \\
\hline & & luinc & 0 & 20 & $1.01 \mathrm{E}-08$ & \\
\hline & & choline & 0 & 27 & $5.29 \mathrm{E}-06$ & \\
\hline & \multirow{3}{*}{ GMRES } & jacobi & 0 & 68 & $9.70 \mathrm{E}-06$ & \\
\hline & & luinc & 0 & 22 & $7.08 \mathrm{E}-06$ & \\
\hline & & choline & 0 & 30 & $1.10 \mathrm{E}-05$ & \\
\hline & \multirow{3}{*}{ QMR } & jacobi & 0 & 89 & $6.43 \mathrm{E}-06$ & \\
\hline & & luinc & 0 & 25 & $2.64 \mathrm{E}-06$ & \\
\hline & & cholinc & 0 & 33 & $9.57 \mathrm{E}-06$ & \\
\hline
\end{tabular}




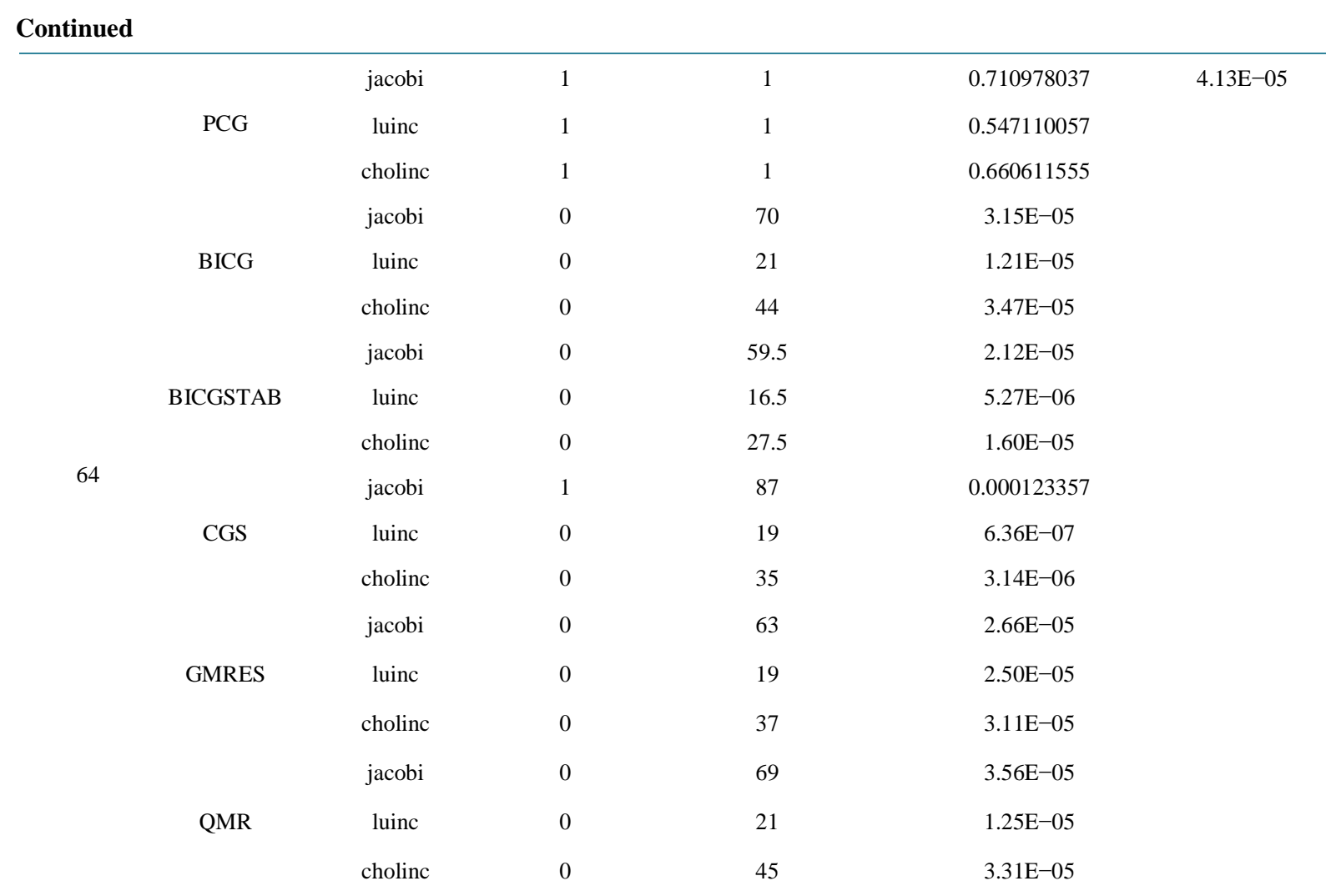

Table 2. Computational details of different fixed point methods.

\begin{tabular}{ccc}
\hline Gamma $=0$ & The fixed point method & No. of iterations \\
\hline & Jacobi & 2246 \\
Gauss-Seidel & SOR & 3124 \\
Backward Gauss-Seidel & 1124 \\
Gamma $=16$ & Backward SOR & 370 \\
& SSOR & 370 \\
& Jacobi & 459 \\
Gauss-Seidel & 231 \\
SOmma $=64$ & 68 \\
& Backward Gauss-Seidel & 231 \\
& Backward SOR & 68 \\
SSOR & 42 \\
Jacobi & 191 \\
Gauss-Seidel & 97 \\
SOckward Gauss-Seidel & 54 \\
Backward SOR & 97 \\
SSOR & 54 \\
& & 20 \\
\hline
\end{tabular}


tral difference method;

- The convergence speed of the six projection methods including PCG, BICG, BICGSTAB, CGS, GMRES and QMR under luinc preconditioning are faster than under cholinc preconditioning, while under cholinc preconditioning are faster than Jacobi preconditioning;

- The six projection methods under Jacobi, luinc and cholinc are convergent when $\gamma=0$, however, for $\gamma=16,64$, the PCG method are not convergent and also the CGS method under Jacobi preconditioning are not convergent when $\gamma=64$.

\section{Acknowledgements}

I thank the editor and the referee for their comments. I would like to express deep gratitude to my supervisor Prof. Dr. Mark A. Peletier whose guidance and support were crucial for the successful completion of this paper. This work was completed with the financial support of Foundation of Guangdong Educational Committee (2014KQNCX161, 2014KQNCX162).

\section{References}

[1] Saad, Y. (2003) Iterative Methods for Sparse Linear Systems. Siam, Bangkok. http://dx.doi.org/10.1137/1.9780898718003

[2] Varga, R.S. (2009) Matrix Iterative Analysis. Volume 27, Springer Science \& Business Media, Heidelberger.

[3] Young, D.M. (2014) Iterative Solution of Large Linear Systems. Elsevier, Amsterdam.

[4] Lanczos, C. (1952) Solution of Systems of Linear Equations by Minimized Iterations. Journal of Research of the National Bureau of Standards, 49, 33-53. http://dx.doi.org/10.6028/jres.049.006

[5] Hestenes, M.R. and Stiefel, E. (1952) Methods of Conjugate Gradients for Solving Linear Systems.

[6] Walker, H.F. (1988) Implementation of the GMRES Method Using Householder Transformations. SIAM Journal on Scientific and Statistical Computing, 9, 152-163. http://dx.doi.org/10.1137/0909010

[7] Sonneveld, P. (1989) CGS, a Fast Lanczos-Type Solver for Nonsymmetric Linear Systems. SIAM Journal on Scientific and Statistical Computing, 10, 36-52. http://dx.doi.org/10.1137/0910004

[8] Freund, R.W. and Nachtigal, N.M. (1991) QMR: A Quasi-Minimal Residual Method for Non-Hermitian Linear Systems. Numerische Mathematik, 60, 315-339. http://dx.doi.org/10.1007/BF01385726

[9] van der Vorst, H.A. (1992) Bi-CGSTAB: A Fast and Smoothly Converging Variant of Bi-CG for the Solution of Nonsymmetric Linear Systems. SIAM Journal on Scientific and Statistical Computing, 13, 631-644. http://dx.doi.org/10.1137/0913035

[10] Brezinski, C., Zaglia, M.R. and Sadok, H. (1992) A Breakdown-Free Lanczos Type Algorithm for Solving Linear Systems. Numerische Mathematik, 63, 29-38. http://dx.doi.org/10.1007/BF01385846

[11] Chan, T.F., Gallopoulos, E., Simoncini, V., Szeto, T. and Tong, C.H. (1994) A Quasi-Minimal Residual Variant of the Bi-CGSTAB Algorithm for Nonsymmetric Systems. SIAM Journal on Scientific Computing, 15, 338-347. http://dx.doi.org/10.1137/0915023

[12] Gutknecht, M.H. (1992) A Completed Theory of the Unsymmetric Lanczos Process and Related Algorithms, Part I. SIAM Journal on Matrix Analysis and Applications, 13, 594-639. http://dx.doi.org/10.1137/0613037

[13] Gutknecht, M.H. (1994) A Completed Theory of the Unsymmetric Lanczos Process and Related Algorithms. Part II. SIAM Journal on Matrix Analysis and Applications, 15, 15-58. http://dx.doi.org/10.1137/S0895479890188803

[14] Eisenstat, S.C. (1981) Efficient Implementation of a Class of Preconditioned Conjugate Gradient Methods. SIAM Journal on Scientific and Statistical Computing, 2, 1-4. http://dx.doi.org/10.1137/0902001

[15] Meijerink, J.V. and van der Vorst, H.A. (1977) An Iterative Solution Method for Linear Systems of Which the Coefficient Matrix Is a Symmetric M-Matrix. Mathematics of Computation, 31, 148-162.

[16] Ortega, J.M. (1988) Efficient Implementations of Certain Iterative Methods. SIAM Journal on Scientific and Statistical Computing, 9, 882-891. http://dx.doi.org/10.1137/0909060

[17] Fowler, A.C. (1997) Mathematical Models in the Applied Sciences. Volume 17, Cambridge University Press, Cambridge. 